\title{
Inflation without Selfreproduction
}

\author{
Viatcheslav Mukhanov \\ Theoretical Physics, Ludwig Maxmillians University,Theresienstr. 37, 80333 Munich, Germany
}

\begin{abstract}
We find a rather unique extension of inflationary scenario which avoids selfreproduction and thus resolves the problems of multiverse, predictability and initial conditions. In this theory the amplitude of the cosmological perturbations is expressed entirely in terms of the total duration of inflation.
\end{abstract}




\section{Introduction}

The recent $\mathrm{CMB}$ observations have unambiguously proven the theory of the quantum origin of the universe structure. According to this theory the initial quantum fluctuations were amplified in the very early universe and provided us with the seeds for galaxies [1, 2]. The simplest mechanism of amplification of these quantum fluctuations is realized in inflationary cosmology according to which the early universe went through the stage of accelerated expansion. On the other hand, the quantum fluctuations amplified during inflation also lead to selfreproduction and do not allow inflation to end up once it was started [6]. Inflation continues forever leading to a metaphysical (non-verifiable) concept of eternal universe and multiverse. According to [3, 4 the multiverse and eternal inflation damage the predictive power of the theory because in this case "anything can happen and will happen an infinite number of times" [5]. Moreover, the most favored by recent observations plateau-like potential returns us back the initial condition problem: "by favoring only plateau-like models, the Planck2013 data creates a serious new challenge for the inflationary paradigm: the universally accepted assumption about initial conditions no longer leads to inflation; instead, inflation can only begin to smooth the universe if the universe is unexpectedly smooth to begin with!" 3. Raising these issues the authors of [3, 4] pose completely legitimate question whether the eternal multiverse inflation, which they call the "postmodern inflationary paradigm" as opposed to "classic inflationary paradigm", does really allow us to explain anything and make any predictions? Surely one could abandon this question restricting oneself only to the last hundred e-folds of inflation responsible for the observable universe and refuse to approximate the theory beyond the scales which we will never see anyway. However, if we want to explain not only the quantum origin of the universe structure, having no alternatives anyway, but also explain the origin of the whole universe we have to understand whether inflation can in principle avoid the problems raised and discussed in [3, 4, [5].

The purpose of this paper is to find a natural extension of inflation to the Planck energy, which avoids the selfreproduction and initial condition problem and thus allows us to turn back to the "classic inflationary paradigm" with all its predictive power.

\section{Inflation and selfreproduction}

Production of a closed universe does not cost any energy because the positive energy of the matter is entirely compensated by the negative energy of the gravitational self-interaction of this matter. Therefore the closed universe can be produced as a result of quantum fluctuations [7. One can expect that quantum fluctuations are essential only at Planck scale and only quantum universes with internal mass of order $10^{-5} \mathrm{~g}$ can easily emerge. If gravity is an attractive force then such universes will immediately recollapse. However, as it was pointed out in $[8,[9,[10$, this does not happen if for some reason the equation of state within 
the Planckian universe corresponds to the cosmological constant $p \approx-\varepsilon$, where $p$ is the pressure and $\varepsilon$ is the energy density. In this case the gravitational field, determined by $\varepsilon+3 p \approx-2 \varepsilon$, is repulsive and instead of collapsing the universe starts to expand with acceleration. As a result the size of the closed universe grows exponentially and its total mass (as well as the number degrees of freedom) also increases exponentially fast. The energy needed to produce the matter comes from the gravitational reservoir with unbounded from below energy. This is a rough picture of the emergence of the causal universe in Minkowski space [8] or from nothing [9], 10] 1 , which was proposed in 80th. This picture is also well supported by quantization of space-time in noncommutative geometry [11]. The stage of accelerated expansion is useful for amplifying quantum fluctuations which later serve as the seeds for galaxy formation [1, 2] and, moreover, it also amplifies the quantum fluctuations of transverse degrees of freedom of the gravitational field (gravitational waves) 12 .

There are many inflationary scenarios in the literature the only purpose of which is to provide us with the stage of the quasi-exponential expansion. These scenarios mainly differ by the choice of a slow-roll scalar field potential "justified" by a "fundamental theory". However such theory is not yet known and hence any particular potential is merely based on the prejudices of the authors. I believe that under such circumstances the more plausible approach is an effective description of inflation in terms of the effective equation of state parametrized by the number of e-folds left until the end of inflation. As it was shown in 13 even the simplest choice for the equation of state allows us to cover nearly all scenarios and to prove that the most valuable predictions of the theory of quantum origin of the universe structure are robust. From the very beginning of the accelerated expansion (inflation) there must be a small deviation of the equation of state from the cosmological constant, i.e.,

$$
1+w \equiv 1+\frac{p}{\varepsilon} \ll 1
$$

but nonvanishing, because otherwise inflation would never end. To describe how this deviation is changing with time we will use as a time parameter the number of e-folds $N$ left to the end of inflation, defined as

$$
a=a_{f} \exp (-N),
$$

where $a$ is the scale factor and $a_{f}$ is its value at the end of inflation when $1+w \simeq O(1)$. The interval of $N$ relevant for the observable universe is not very large, namely, $N<70$. At the end of inflation the squared amplitude of the Newtonian gravitational potential in scales $\lambda$ is (see [14])

$$
\left.\Phi_{\lambda}^{2} \simeq \frac{\varepsilon}{1+w}\right|_{N_{\lambda}}
$$

\footnotetext{
${ }^{1}$ The idea of the creation of universe from nothing was suggested independently by $\mathrm{L}$. Grishchuk, Y. Zeldovich [9] and A. Vilenkin[10], who also offered the mathematical description for the nucleation process.
} 
where we have used the Planck units in which $\varepsilon_{P l}=1$ and $N_{\lambda}$ is the number of e-folds left to the end of inflation since the time when the commoving scale $\lambda$ crosses the Hubble scale $H^{-1}=a / \dot{a}$, that is,

$$
H^{-1}=a_{f} e^{-N_{\lambda}} \lambda \text {. }
$$

For observed in the CMB experiments scales $N_{\lambda} \simeq 50 \div 60$. Assuming that the equation of state is monotonic and smooth and taking into account that $1+w \simeq O(1)$ when inflation ends at $N \simeq 1$, it is rather natural to approximate it for $N>1$ as

$$
1+w \simeq \frac{\beta}{N^{\alpha}}
$$

where $\alpha$ and $\beta$ are both the positive constants of order unity. Using the energy conservation equation

$$
\dot{\varepsilon}=-3 H(\varepsilon+p),
$$

rewritten as

$$
\frac{d \ln \varepsilon}{d N}=3(1+w) \simeq \frac{3 \beta}{N^{\alpha}},
$$

we find

$$
\varepsilon(N) \simeq \begin{cases}\varepsilon_{0} N^{3 \beta}, & \alpha=1, \\ \varepsilon_{0} \exp \left(-\frac{3 \beta}{\alpha-1} \frac{1}{N^{\alpha-1}}\right), & \alpha \neq 1 .\end{cases}
$$

Correspondingly the amplitude of the scalar perturbations is

$$
\Phi_{\lambda}^{2} \simeq \begin{cases}\varepsilon_{0} N^{3 \beta+1}, & \alpha=1, \\ \varepsilon_{0} N^{\alpha} \exp \left(-\frac{3 \beta}{\alpha-1} \frac{1}{N^{\alpha-1}}\right), & \alpha \neq 1 .\end{cases}
$$

The spectral index $n_{s}$, defined in the observable range of scales via $\Phi^{2} \propto \lambda^{1-n_{s}}$, is then equal to

$$
1-n_{s}=\frac{d \ln \Phi^{2}}{d \ln \lambda}=\frac{d \ln \Phi^{2}}{d N_{\lambda}} \simeq \begin{cases}\frac{3 \beta+1}{N_{\lambda}}, & \alpha=1 \\ \frac{\alpha}{N_{\lambda}}, & \alpha>1 .\end{cases}
$$

It was shown in [13] that in terms of the slow-roll scalar field potentials the case $\alpha=1$ corresponds to the chaotic inflation [16] with power-law potentials, $\alpha=2$ describes the plateau-like potentials and finally $\alpha>2$ describe the scenarios with small scalar field, in particular, the new inflation [17. According to the recent Planck data [15], $n_{s}=0.96 \pm 0.007$. Therefore the cases $\alpha=1$ for $3 \beta=1$ and $\alpha=2$ are strongly favored by the observations. The energy density at the end of inflation, $\varepsilon_{0}$ in (8), is determined by requiring $\Phi_{\lambda}^{2} \simeq 10^{-9}$ for $N_{\lambda} \simeq 50$ to fit the observations. For both cases above we have,

$$
\varepsilon_{0} \simeq 10^{-12},
$$

in Planck units. The models with $\alpha=1,3 \beta=1$ and $\alpha=2$ are indistinguishable from the point of view of the scalar perturbations because they result in precisely 
the same spectrum of inhomogeneities. However, they predict different ratios of tensor to scalar perturbations (see [14]):

$$
r=24(1+w)=\frac{24 \beta}{N^{\alpha}} .
$$

In case $3 \beta=1$ we have $r=0.16$. So high value of $r$ is seriously disfavored by the Planck data according to which $r<0.11$ at $95 \%$ confidence level [15]. For $\alpha=2$ the amount of gravitational waves is about $N_{\lambda} \simeq 50$ times less and corresponds to $r=0.003$ in perfect agreement with observations. Thus, the recent CMB Planck results seems strongly favor plateau-like potentials and rule out at significant confidence level both the chaotic and new inflation.

As we have seen above, given the spectral index $n_{s}=0.96$ one can fix the energy scale at the end of inflation to be about $\varepsilon_{0} \simeq 10^{-12}$. In turn this determines the lower bound on the amount of the gravitational waves. Hence, taking into account the results of the CMB observations we conclude that $r=O(1) \times 0.003$ characterizes not only the most likely expected level of the gravitational waves but also it is the lower bound on the amount of the tensor perturbations produced during inflation.

It follows from (9) that the amplitude of perturbations on the scales which left the Hubble scale at

$$
N>N_{s r} \simeq \begin{cases}\varepsilon_{0}^{-\frac{1}{3 \beta+1}}, & \alpha=1, \\ \varepsilon_{0}^{-\frac{1}{\alpha}}, & \alpha>1 .\end{cases}
$$

exceeds unity and the universe on these scales should be very inhomogeneous. This happens because for $N>N_{s r}$ the universe is in the selfreproduction regime in which quantum fluctuations dominate over the classical evolution and the energy density in the patches of Hubble size instead of decreasing can increase as a consequence of the quantum jumps. As a result, there emerges exponentially growing volume, where inflation never ends and finally this leads to eternal infinite multiverse dominated by inflating regions. In the cases $\alpha=1,3 \beta=1$ and $\alpha=2$ the minimal number of e-folds which leads to the eternal inflation is rather large, $N_{s r} \simeq 10^{6}$, compared to the number of e-folds $N \simeq 10^{2}$ needed to explain the observable universe. One, of course, could doubt the applicability of the theory so far beyond the observable scales. However, if inflation pretends that it really solves the initial condition problem for the whole universe as it was claimed in the "classic inflationary paradigm", one has either to resolve the measure problem in the multiverse (and it is not even clear whether this problem can be well formulated) or find a way to avoid the multiverse and eternity, thus escaping the problems of infinities and measures. 


\section{$3 \quad$ Avoiding selfreproduction}

At first glance it looks like the necessary and sufficient condition for the selfreproduction,

$$
\left.\frac{\varepsilon}{1+w}\right|_{N_{s r}} \simeq 1
$$

is always satisfied for some $N_{s r}$ especially if inflation begins at $\varepsilon \simeq 1$ to avoid the initial condition problem. In fact, for the models considered above $1+w$ decreases with $N$ while $\varepsilon(N)$ either grows $(\alpha=1)$ or approaches a constant value $(\alpha>1)$. In this case equation (14) always has a solution at $\varepsilon\left(N_{s r}\right)<1$ and the selfreproduction is unavoidable. If we assume that for large $N$ the equation of state approaches constant value, $1+w_{0} \neq 0$, then the energy density would grow with $N$ and at $\varepsilon_{s r} \simeq 1+w_{0} \ll 1$ we again inevitably enter the selfreproduction regime. Therefore, the only way to avoid this regime is to assume that at some large $N$, the value of $1+w(N)$ begins to increase with $N$. As it follows from (7) the energy density is then equal to the Planck density $\varepsilon \simeq 1$ for some finite $N_{m}$. Thus, if we want to avoid the selfreproduction and do not face the problem of initial conditions the required equation of state must simultaneously satisfy the following requirements:

a) $1+w(N) \simeq 1$ at $N \simeq 1$ (to have graceful exit),

b) $1+w(N) \leq 2 / 3$ at $N \simeq N_{m}$ (to solve initial condition problem),

c) $1+w(N) \ll 1$ for $1<N<N_{m}$ (inflation),

d) $1+w(N)>\varepsilon(N)$ for $1<N<N_{m}$ (no selfreproduction).

It is clear that if $1+w \ll 1$ at $\varepsilon \simeq 1$ then the selfreproduction would take place already at the energy density $\varepsilon \simeq 1+w \ll 1$. Therefore, we assume that at Planck scale $1+w\left(N_{m}\right)$ is for instance $1 / 3$, just to begin the accelerated expansion and $1+w \ll 1$ after that. The conditions b)-c) can simultaneously be satisfied if we assume that

$$
1+w \simeq \varepsilon^{\gamma}
$$

with $\gamma<1$. In fact, in this case $1+w \ll 1$ for $\varepsilon \ll 1$ and hence inflation takes place; moreover,

$$
\frac{\varepsilon}{1+w} \simeq \varepsilon^{1-\gamma}<1
$$

for $\varepsilon<1$, and "no selfreproduction" condition d) is also satisfied. Substituting (15) in (77) and integrating we find

$$
\varepsilon(N) \simeq\left(\frac{1}{3 \gamma\left(N_{m}+1-N\right)}\right)^{1 / \gamma}
$$

and

$$
1+w(N) \simeq \frac{1}{3 \gamma\left(N_{m}+1-N\right)} .
$$

At the beginning of inflation, at $N=N_{m}$, both $\varepsilon$ and $1+w$ are of order unity and for $N<N_{m}$ we have inflation with $1+w \simeq\left(3 \gamma N_{m}\right)^{-1} \ll 1$. One can wonder 
to what extent the choice (15) is ambiguous. Let us show that it is nearly unique. With this purpose we consider more general function for $1+w(N)$ as, for example,

$$
1+w(N) \simeq \frac{a}{\left(N_{m}+1-N\right)^{\delta}}
$$

with $\delta \neq 1$. Then we find

$$
\varepsilon(N) \simeq \exp \left(\frac{3 a}{\delta-1} \frac{1}{\left(N_{m}+1-N\right)^{\delta-1}}\right),
$$

where the constant of integration is fixed by imposing $\varepsilon\left(N_{m}\right) \simeq 1$ to avoid the initial condition problem. It is clear that for $\delta>1$ the energy density is of order unity for $N<N_{m}$, while $1+w \ll 1$, and hence the selfreproduction is inevitable. For $\delta<1$ the energy density drops too fast and for instance, for $\delta=1 / 2$ it is about $\varepsilon(N) \simeq \exp \left(-N_{m}^{1 / 2}\right)$ at $N \simeq N_{m} / 2$. Therefore the amplitude of perturbations generated at inflation will be too small to explain the structure of the universe. In fact, to be in agreement with observations $N_{m}^{1 / 2}$ must be larger than, at least, hundred and hence $\varepsilon \simeq \exp (-100)$ is much less compared to the required $10^{-12}$. Thus, the behavior of the equation of state given by (18) is nearly unique at large $N$. However, it does not satisfies the condition a) above. In fact, according to (18) $1+w$ approaches the constant for small $N$ and inflation has no graceful exit. Therefore (18) should be modified for small $N$. This can be easily achieved by combining (5) with (18) and it is obvious that

$$
1+w(N) \simeq \frac{\beta}{N^{\alpha}}+\frac{1}{3 \gamma\left(N_{m}+1-N\right)},
$$

satisfies all conditions a)-d) simultaneously. In this case $1+w$ is of order unity at the beginning of inflation at Planck density, then it decreases, reaches the minimum and begins to grow towards the end of inflation and finally becomes of order unity at $N \simeq 1$. The energy density is given by

$$
\varepsilon(N) \simeq \begin{cases}\frac{1}{\left(N_{m}+1-N\right)^{1 / \gamma}}\left(\frac{N}{N_{m}}\right)^{3 \beta}, & \alpha=1, \\ \frac{1}{\left(N_{m}+1-N\right)^{1 / \gamma}} \exp \left(-\frac{3 \beta}{\alpha-1} \frac{1}{N^{\alpha-1}}\right), & \alpha \neq 1,\end{cases}
$$

where the constant of integration is fixed by requiring $\varepsilon\left(N_{m}\right) \simeq 1$.

In the case $\alpha=1$ and $3 \beta=1$, the amplitude of scalar perturbations is about

$$
\Phi^{2} \simeq \frac{1}{\left(N_{m}-N\right)^{\frac{1}{\gamma}-1}}\left(\frac{N}{N_{m}}\right)^{2}
$$

and correspondingly the spectral index is

$$
1-n_{s} \simeq \frac{2}{N}+\left(\frac{1}{\gamma}-1\right) \frac{1}{N_{m}-N} .
$$


As we see in non-eternal inflating universe the amplitude of perturbations is entirely determined by the duration of inflation and has the required value if

$$
N_{m}^{\frac{1}{\gamma}+1} \simeq 10^{12} .
$$

Because $\gamma<1$ (see (15)) the maximal duration of inflation cannot exceed $10^{6}$ e-folds. For $1 / \gamma=2$ the maximal number of e-folds, which determines the size of the universe, is about $10^{4}$. Because the number of e-folds should definitely be larger that $10^{2}$ we find that $1 / \gamma$ must be smaller than 5 . The model above describes inflation which begins at Planck density (hence no initial condition problem) and creates one unique universe (avoiding the selfreproduction and multiverse problems). However, this model is disfavored by the CMB observations because it produces too much gravitational waves. The most favorable (nearly unique) model, which is in agreement with the observations, corresponds to $\alpha=2$. In this case

$$
\Phi^{2} \simeq \frac{1}{\left(N_{m}-N\right)^{\frac{1}{\gamma}-1}} \frac{N^{2}}{N_{m}+N^{2}},
$$

and

$$
1-n_{s} \simeq \frac{2}{N}\left(1+\frac{N^{2}}{N_{m}}\right)^{-1}+\left(\frac{1}{\gamma}-1\right) \frac{1}{N_{m}-N} .
$$

Here the duration of inflation is determined by the condition

$$
N_{m}^{\frac{1}{\gamma}} \simeq 10^{12},
$$

and hence for $1 / \gamma=2$ the maximal number of e-folds is $10^{6}$ which would be about the scale of selfreproduction if $1+w$ is given by (5). However, in our case the second term in (21) is larger than the first one for $N>N_{m}^{1 / 2} \simeq 10^{3}$ and hence it determines the evolution for $N>10^{3}$. For $1 / \gamma=4$, we obtain $N_{m} \simeq 10^{3}$ and the spectral index would be substantially corrected compared to the case (5) in observable scales at $N \simeq 50$. Thus, we conclude that $1 / \gamma$ must be 2 or 3 .

\section{Slow-roll potentials}

In the modern literature the "playground of inflationary scenarios" mainly consists of the slow-roll potentials which sometimes are rather complicated and based on ugly constructions. The only purpose of these constructions is to justify the quasi-exponential expansion. Moreover, the only relevant for comparison with observations features of the potential are its value and its first and second derivatives basically at one point. Thus forgetting the questionable justification of the scenarios from the point of view of a "fundamental theory", the scalar field potentials are not more than merely the way to parametrize the missing knowledge about fundamental physics at high energies, not better than 
parametrization above. I believe that the scalar field parametrization is not so natural as it looks at the first glance and not even so convenient as used above. Therefore, perhaps it is not surprising that all original scenarios which look simple in terms of the scalar field have failed to describe the observations. In fact, everything we need for explaining the observations is the description of inflation in terms of some effective equation of state, because gravity seems entirely ignores the fundamental physics behind the equation of state. However, with the purpose to make connection with the current literature I will reformulate the results above in terms of the slow-roll potentials.

To determine the potential $V(\varphi) \simeq \varepsilon$ we have to express $N$ in terms of the scalar field $\varphi$. With this purpose we write

$$
\frac{d \varphi}{d N}=\frac{\dot{\varphi}}{-H}=\sqrt{\frac{3}{8 \pi}(1+w),}
$$

where we have taken into account that $\varepsilon+p=\dot{\varphi}^{2}$ and $H^{2}=8 \pi \varepsilon / 3$ (in the Planck units). Substituting here (21) and integrating the resulting equation we find than for $\alpha=1$,

$$
\varphi \simeq \begin{cases}\sqrt{N}, & N_{m}-N>N \\ \varphi_{m}-\sqrt{N_{m}-N}, & N_{m}-N<N\end{cases}
$$

Taking this into account in expression (22) we find that the even in $\varphi$ potential which well approximates the required behavior of the equation of state is

$$
V(\varphi) \simeq \frac{\varphi_{m}^{a}\left(\varphi / \varphi_{m}\right)^{2}}{\left(\varphi_{m}^{2}-\varphi^{2}\right)^{a}}
$$

where $a=2 / \gamma$ and can be for instance $3,4 \ldots$ In fact, for positive $\varphi$

$$
1+w \simeq\left(\frac{V_{, \varphi}}{V}\right)^{2} \simeq \frac{1}{\varphi^{2}}\left(\frac{\varphi_{m}}{\varphi_{m}-\varphi}\right)^{2},
$$

and inflation takes place for $1<\varphi<\varphi_{m}-1$. The spectrum of perturbations is given by

$$
\Phi \simeq \frac{V^{3 / 2}}{V_{, \varphi}} \simeq \frac{\left(\varphi / \varphi_{m}\right)^{2}}{\left(\varphi_{m}-\varphi\right)^{\frac{a}{2}-1}}
$$

and $\Phi$ becomes of order unity only at Planck scale when $\varphi_{m}-\varphi \simeq 1$. To get the required amplitude of perturbations in galactic scales we have to take

$$
\varphi_{m} \simeq 10^{\frac{12}{a+2}}
$$

For $a=4$ we have $\varphi_{m} \simeq 10^{2}$ and correspondingly the total number of e-folds on inflation is $N_{m} \simeq \varphi_{m}^{2} \simeq 10^{4}$. As we have noticed above the potential (31) predicts too much gravitational waves.

More realistic potential, which is in perfect agreement with the observations, is

$$
V(\varphi) \simeq \frac{[1-\exp (-\varphi)]^{2}}{\left(\varphi_{m}-\varphi\right)^{a}}
$$


For this potential

$$
1+w \simeq\left(\frac{V_{, \varphi}}{V}\right)^{2} \simeq\left(\frac{a}{\varphi_{m}-\varphi}+2 e^{-\varphi}\right)^{2},
$$

and

$$
\Phi \simeq \frac{V^{3 / 2}}{V_{, \varphi}} \simeq \frac{1}{\left(\varphi_{m}-\varphi\right)^{\frac{a}{2}-1}\left(a+2 e^{-\varphi} \varphi_{m}\right)}
$$

for $1<\varphi<\varphi_{m}$. The value of $\varphi_{m}$ is entirely fixed by the required amplitude of perturbations and is equal to $\varphi_{m} \simeq 10^{\frac{12}{a}}$. The possible values for $a$ are 4 and 6. For $\varphi<\ln \varphi_{m}$ we obtain from (29) $N \simeq e^{\varphi}$, while for $\varphi>\ln \varphi_{m}$ one has $N_{m}-N \simeq\left(\varphi_{m}-\varphi\right)^{2}$. Therefore this model is similar to the model (21) with $\alpha=2$ and the potential (35) is of type which is the best from the point of view of the CMB observations and at the same time does not lead to the problems of initial conditions and multiverse, thus recovering the "classic inflationary paradigm".

\section{Discussion}

The Planck measurements have unambiguously confirmed the main predictions of the theory of quantum origin of the universe structure. Namely, the adiabatic nature and the Gaussian origin of primordial perturbations were established beyond any reasonable doubt. Even more amazing, more nontrivial infrared logarithmic tilt of the spectrum, first predicted in [2, was discovered at 6 sigma confidence level. The simplest way to amplify the quantum fluctuations is provided by the stage of inflation. Although nobody doubt the quantum origin of the primordial fluctuations, there are still claims in the literature that basically the same mechanism of amplification of quantum fluctuations can work also either in a bouncing universe on the stage of super slow contraction [18] or in conformal rolling scenario [19. The generated spectra in the alternative theories are not the predictions of the theory, but rather postdictions which are constructed to be in agreement with observations. Nevertheless, this is not enough to rule out these possibilities at the level of a "theorem". Thus, at the moment the only robustly established experimental fact is the quantum origin of the universe structure with a little uncertainty left for the mechanism of amplification of quantum fluctuations. To firmly establish that namely inflation has provided us this mechanism one has to find the primordial gravitational waves the lower bound on which for the spectral index $n_{s}=0.96$ corresponds to $r$ about 0.003 . According to [3, 4] one of the main motivations for looking the alternatives to inflation is the failure of predictability of so called "postmodern inflationary paradigm". Paradoxically this trouble seems to be due to the same successful quantum fluctuations with the red-tilted spectrum which lead to the galaxies. On one hand the quantum fluctuations explain the observed large scale structure of the universe, but on the other hand they are also responsible for the selfreproduction and produce eternal inflating multiverse where "anything 
can happen and will happen an infinite number of times" [5]. In this paper I have shown how this problem can be avoided. Using the effective description of inflation I have found nearly unambiguous extension of inflation which avoids the selfreproduction. What is yet missing in this description is a justification of the model from the point of view of some fundamental theory. However, under circumstances when only effective description of inflation is needed to explain the observations and there are no even slightest experimental hints how the fundamental theory should look like at very high energies such an approach looks as the most plausible. Moreover, it can provide us with hints about fundamental theory, which can avoid even metaphysical problems.

Acknowledgements This work was supported by TRR 33 "The Dark Universe" and the Cluster of Excellence EXC 153 "Origin and Structure of the Universe".

\section{References}

[1] G. V. Chibisov and V. F. Mukhanov, Galaxy Formation and Phonons, Lebedev Phys. Inst. Preprint No. 162, 1980; MNRAS vol. 200, pp. 535550,1982 .

[2] V. F. Mukhanov and G. V. Chibisov, Quantum fluctuation and nonsingular Universe, JETP Letters, vol. 33, pp. 532-535, 1981.

[3] A. Ijjasa, P. J. Steinhardt and A. Loeb, Inflationary paradigm in trouble after Planck2013, Phys.Lett. B723, pp. 261-266, 2013.

[4] A. Ijjasa, P. J. Steinhardt and A. Loeb, Inflationary schism after Planck2013, e-Print: arXiv:1402.6980, 2014.

[5] A. H. Guth, D. I. Kaiser, and Y. Nomura, Inflationary paradigm after Planck 2013, arXiv:1312.7619, 2013.

[6] A. Vilenkin, Birth of inflationary universes, Phys.Rev. D27, 2848, 1983

[7] E. P. Tryon, Nature, 246, 396, 1973.

[8] R. Brout, F. Englert, and E. Gunzig, The Causal Universe, General Relativity and Gravitation, vol. 10, no. 1, pp. 1-6, 1979.

[9] L. Grischuk, Za. B. Zeldovich, Complete Cosmological Theories, In *Moscow 1981, Proceedings, Quantum Gravity*, 71-85, 1981

[10] A. Vilenkin, Creation of the Universes from Nothing, Phys. Lett. 117B, 25,1982 .

[11] A. Chamseddine, A. Connes and V. Mukhanov, Quanta of Geometry, to be published. 
[12] A. Starobinsky, Spectrum of Relict Gravitational Radiation and the Early State of the Universe, JETP Lett. vol. 30, pp. 682-685, 1979.

[13] V. Mukhanov, Quantum Cosmological Perturbations: Predictions and Observations, Eur.Phys.J. C73, 2486, 2013.

[14] V. Mukhanov, Physical Foundations of Cosmology, Cambridge University Press, Cambridge, UK, 2005.

[15] Planck Collaboration: Planck 2013 results. XXII. Constraints on inflation, arXiv:1303.5082, 2013.

[16] A. Linde, Chaotic Inflation, Phys. Lett. B, vol. 129, pp. 177-180, 1983.

[17] A. D. Linde, Phys.Lett. B108, 389, 1982; A. Albrecht and P. J. Steinhardt, Phys.Rev.Lett. 48, 1220, 1982.

[18] see A. Ijjasa, P. J. Steinhardt and A. Loeb, Scale-free primordial cosmology, Phys.Rev. D89, 023525, 2014 and references there.

[19] M. Libanov, S. Ramazanov and V. Rubakov, Scalar perturbations in conformal rolling scenario with intermediate stage, JCAP 1106, 010, 2011; S. A. Mironov, S. R. Ramazanov, V. A. Rubakov, Effect of intermediate Minkowskian evolution on CMB bispectrum, JCAP 1404, 015, 2014. 